MITSUBISHI ELECTRIC RESEARCH LABORATORIES

http://www.merl.com

\title{
Battery Energy Management in Heterogeneous Wireless Machine-to-Machine Networks
}

\author{
Liu, K.; Guo, J.; Orlik, P.V.; Parsons, K.; Sawa, K. \\ TR2015-101 September 2015
}

\begin{abstract}
The IETF standardized the IPv6 Routing Protocol for Low-Power and Lossy Networks (RPL) to meet routing requirements of the emerging applications. RPL is a distributed routing protocol and shows good scalability and fast network setup. However, RPL does not support sleep operation well. To provide efficient energy management and enhance RPL for sleep operation support, this paper presents battery energy management solutions for heterogeneous wireless machine-to-machine networks containing both battery powered nodes and mains powered nodes. We introduce a distributed sleep model for battery powered nodes to manage their own sleep schedules based on their internal parameters and observed network conditions. We propose two broadcast message delivery methods for battery operated networks that use distributed sleep control. Two battery node aware routing metrics are introduced to discover more battery energy efficient routes. We also present a battery energy efficient routing protocol called B-RPL to leverage distributed sleep model and introduced routing metrics. A battery energy efficient data packet transmission and forwarding method is provided to select the most battery energy efficient route among multiple active routes to transmit and forward data packets. Simulation results show that compared with standard RPL, the proposed B-RPL can extend network lifetime by two times and improve data packet delivery rate by $75 \%$.
\end{abstract}

2015 IEEE VTC2015-Fall

This work may not be copied or reproduced in whole or in part for any commercial purpose. Permission to copy in whole or in part without payment of fee is granted for nonprofit educational and research purposes provided that all such whole or partial copies include the following: a notice that such copying is by permission of Mitsubishi Electric Research Laboratories, Inc.; an acknowledgment of the authors and individual contributions to the work; and all applicable portions of the copyright notice. Copying, reproduction, or republishing for any other purpose shall require a license with payment of fee to Mitsubishi Electric Research Laboratories, Inc. All rights reserved.

Copyright (c) Mitsubishi Electric Research Laboratories, Inc., 2015

201 Broadway, Cambridge, Massachusetts 02139 



\section{Battery Energy Management in Heterogeneous Wireless Machine-to-Machine Networks}

\author{
Kaikai Liu \\ Scalable Software Systems Lab \\ University of Florida \\ kaikailiu@ufl.edu
}

\author{
Jianlin Guo, Philip Orlik, Kieran Parsons \\ Mitsubishi Electric Research Laboratories \\ Cambridge, MA 02139, USA \\ \{guo, porlik, parsons\}@merl.com
}

\author{
Kentaro Sawa \\ Mitsubishi Electric Corporation IT R\&D Center \\ Ofuna, Kamakura, Kanagawa 247-8501, Japan \\ Sawa.Kentaro@bk.MitsubishiElectric.co.jp
}

\begin{abstract}
The IETF standardized the IPv6 Routing Protocol for Low-Power and Lossy Networks (RPL) to meet routing requirements of the emerging applications. RPL is a distributed routing protocol and shows good scalability and fast network setup. However, RPL does not support sleep operation well. To provide efficient energy management and enhance RPL for sleep operation support, this paper presents battery energy management solutions for heterogeneous wireless machine-tomachine networks containing both battery powered nodes and mains powered nodes. We introduce a distributed sleep model for battery powered nodes to manage their own sleep schedules based on their internal parameters and observed network conditions. We propose two broadcast message delivery methods for battery operated networks that use distributed sleep control. Two battery node aware routing metrics are introduced to discover more battery energy efficient routes. We also present a battery energy efficient routing protocol called B-RPL to leverage distributed sleep model and introduced routing metrics. A battery energy efficient data packet transmission and forwarding method is provided to select the most battery energy efficient route among multiple active routes to transmit and forward data packets. Simulation results show that compared with standard RPL, the proposed B-RPL can extend network lifetime by two times and improve data packet delivery rate by $75 \%$.
\end{abstract}

Keywords-Energy aware, battery operated networks, heterogeneous M2M, self sleep control, idle time reduction, battery node aware routing, overhearing minimization, energy efficient data delivery.

\section{INTRODUCTION}

The large scale self-organizing wireless machine-tomachine (M2M) networks are being driven by emerging applications in the areas of the Internet of Things (IoT), smart grid, industrial automation, building and home automation. Unlike conventional wireless sensor networks, wireless M2M networks are typically heterogeneous such that networks contain nodes with different resources and capabilities. As a result, network technologies such as routing algorithms have to consider this heterogeneity. This paper focuses on power source heterogeneity. We study heterogeneous wireless M2M networks containing both battery powered (BP) nodes and mains powered (MP) nodes.

A node consumes its energy for data packet transmission, data packet receiving, control message transmission and control message receiving. These categories of energy consumption are considered as necessary energy use. However, a

This work was done while Kaikai Liu was an intern at Mitsubishi Electric Research Laboratories (MERL). node also consumes its energy on idle listening, overhearing, collision, etc. These categories of energy consumption are considered as energy waste. Our objective is the development of energy saving solutions to minimize the energy waste on BP nodes.

We define the network lifetime as the operation time of the BP node that first runs out of battery power. How to save battery energy and prolong network lifetime is critical. Energy saving technologies can be categorized into topology control algorithms and sleep management algorithms. Topology control algorithms focus on transmission power control such that data are only transmitted to the destined nodes. A comprehensive survey on the distributed topology control techniques to extend the lifetime of battery powered wireless sensor networks can be found in [1]. Topology control technology, however, does not reduce energy consumed on idle listening and may degrade network capacity. Sleep management algorithms schedule nodes to enter sleep mode to save energy. In sleep mode, a node consumes much less energy than idle mode because node's radio is off and its processor is in a low power state.

This paper proposes sleep management solutions to save battery energy and extend network lifetime for large scale heterogeneous wireless M2M networks containing both BP nodes and MP nodes.

The rest of this paper is organized as follows. Section II overviews related work. Section III describes the distributed sleep model. Section IV proposes two broadcast message delivery methods. Section V introduces two battery node aware routing metrics. Details of battery energy efficient routing protocol (B-RPL) are presented in Section VI. Section VII provides B-RPL performance evaluation and analysis based on simulations. We conclude our work in Section VIII.

\section{RELATED WORKS}

In networks containing BP nodes, extending network lifetime is one of key issues to be addressed. Routing plays a crucial role in energy saving. [2] proposed a cluster based energy efficient routing protocol for wireless sensor networks so that the cluster head manages sleep schedules of sensor nodes based on the node density. Various other technologies have also been proposed to extend the lifetime of battery powered wireless sensor networks. However, these existing energy saving solutions are designed for homogeneous wireless sensor networks and do not fit heterogeneous wireless M2M networks well. For example, it is not beneficial to forward 
packets exclusively to a MP node if the route via the MP node contains a very low BP node.

The RPL is an IPv6 based routing protocol designed by Internet Engineering Task Force (IETF) and standardized as RFC 6550 [3]. RPL organizes nodes in a network as a Destination Oriented Directed Acyclic Graph (DODAG). A DODAG contains one sink node called the DODAG root. To build upward routes from nodes to the sink node, the sink node initiates the DODAG Information Object (DIO) message propagation. A DIO message contains information to construct a DODAG. As DIO message propagates, the DODAG topology is constructed. RPL allows a node to select multiple parents. One of the parents is selected as the preferred parent and others as backup parents. The preferred parent is used as default next hop for upward packet transmission. RPL uses Destination Advertisement Object (DAO) message to construct downward routes from the sink node to other nodes. The DODAG Information Solicitation (DIS) message is used to solicit a DIO from a RPL node.

RPL shows good scalability and fast network set up. However, RPL may suffer from severe unreliability [4]. In addition, RPL does not provide any method to support sleep nodes. Several techniques have been proposed to enhance the reliability of RPL. [5] proposed a multipath opportunistic forwarding scheme to extend RPL with the possibility of forwarding packets over multiple routes. [6] proposed a stability metric based mechanism for RPL to improve the reliability. Again, these enhancements are made for homogeneous wireless networks without considering sleep operation. In this paper, we propose new technologies to enhance RPL for heterogeneous wireless M2M networks containing both BP nodes and MP nodes.

\section{DistRibuted SleEP MOdEL}

The centralized sleep control is impractical for large scale wireless networks with multihop communications. We propose a distributed sleep model such that each BP node manages its own sleep schedules. As illustrated in Fig. 1, a sleep interval (SI) begins with an active period (AP) followed by a sleep period (SP). When a BP node wakes up from sleep, it transmits a short wakeup message to announce it is awake and ready to receive packets. When a $\mathrm{BP}$ node enters the $\mathrm{SP}$, its radio is turned off. A BP node dynamically determines its AP and SP lengths based on its internal parameters and observed network conditions. Equation (1) shows a method to compute the AP length by a BP node using its buffer size $(B S)$, number of buffered packets $(N o B P)$, residual battery percentage $(R B P)$ and observed percentage of the BP neighbors $(P o B P N)$, subtree size $(S S)$ and network size $(N S)$.

$$
|A P|=C_{A P} \times \frac{N o B P}{B S} \times R B P \times P o B P N \times \frac{S S}{N S} \times|S I|
$$

where $C_{A P}$ is a positive coefficient to scale $|A P|$ under the condition $|A P|<|S I|$. Based on equation (1), a BP node sets a longer AP if it has more buffered packets, higher battery level, more battery powered neighbors and a larger sub-tree. A BP node may extend its AP at the end of current AP. A BP node may indicate how long it is going to be active in wakeup message. However, a BP node can not set its AP length to zero. Therefore, a minimum AP length threshold $A P_{\text {min }}$ is defined for a network. If the $|A P|$ calculated in equation (1) is less than $A P_{\text {min }}$, a BP node set its $|A P|$ to $A P_{\text {min }}$. Similarly, a $\mathrm{BP}$ node can not set its SP length infinitely long. Likewise, a maximum SP length threshold $S P_{\max }$ is also defined for a network. The $N S, A P_{\min }$ and $S P_{\max }$ can be configured on each BP node or carried in a control message such as DIO message in RPL.

Different BP nodes such as $B_{1}, B_{2}$ and $B_{3}$ have different sleep intervals. If a BP node has no data to transmit, it schedules periodic sleep intervals. If a node (mains powered or battery powered) such as $B_{2}$ has data to send to a BP node such as $B_{3}$, it must make sure the battery powered receiver such as $B_{3}$ is awake. The wakeup message is an awake indication in this case. The MP nodes do not sleep and are always active. Thus a node sending a packet to a MP node does not wait for a wakeup message.

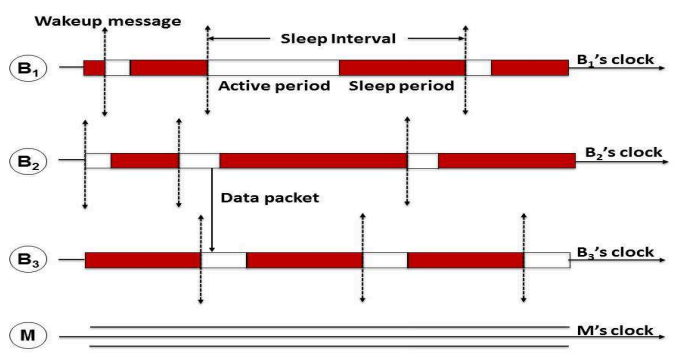

Fig. 1: Distributed Sleep Model Illustration

When using a distributed sleep model, the idle listening time spent waiting for a wakeup message could be long. Therefore, a BP node must efficiently manage its sleep schedule to reduce idle listening time. Two approaches are introduced to minimize the idle time: overhearing and dynamic wakeup. Overhearing is a way to reduce idle listening time. If a sender overhears any message from receiver, the receiver is awake. Therefore, a sender can send packets and go to sleep. Dynamic wakeup message is another method to reduce idle listening time. While a BP sender is waiting for a wakeup message from its receiver, it can send a dynamic wakeup message to let its neighbors know it is awake. A BP node can also send a dynamic wakeup message if it extends its current AP.

\section{Broadcast Message DeliVery Using DisTRIBUTED SLEEP MODEL}

To build and maintain a network, some broadcast messages such as DIO message in RPL must be delivered to all nodes. In other words, a node must deliver important broadcast messages to all neighbors. However, due to the unmanaged sleep schedules of the distributed sleep model, broadcast message delivery becomes a challenge. We propose two broadcast message delivery methods.

One method to deliver a broadcast message to all neighbors is to transmit the broadcast message for $N_{B C S T}=$ $\operatorname{ceil}\left(\left(A P_{\min }+S P_{\max }\right) / A P_{\min }\right)+1$ times. These $N_{B C S T}$ broadcasts can be uniformly distributed over a time period called broadcast interval with the length equal to $A P_{\min }+$ $S P_{\max }$. Because time between two consecutive broadcasts is less than $A P_{\text {min }}$ and every $\mathrm{BP}$ node has an $\mathrm{AP}$ with $|A P| \geq A P_{\min }$ and a SP with $|S P| \leq S P_{\max }$, these $N_{B C S T}$ 
broadcasts guarantee every neighbor receives at least one copy of a broadcast message. Between two consecutive broadcasts, the transmitting node can go to sleep. This broadcast message delivery method is more efficient if the $A P_{\min }$ is relatively large so that $N_{B C S T}$ is relatively small.

Another method to deliver a broadcast message to all neighbors is to have the transmitting node remain awake for a broadcast interval. At the beginning of the broadcast interval, the node transmits the broadcast message once to make sure all MP neighbors receive the broadcast message. For the remainder of the broadcast interval, the node transmits the broadcast message once every time it receives a wakeup message from a BP neighbor. With this method, transmitting node transmits the broadcast message for number of BP neighbors $(N o B P N)$ plus one times. This broadcast message delivery method is more efficient if transmitting node has fewer BP neighbors.

\section{BAtTery Node Aware Routing Metrics}

RPL defines routing metrics in RFC 6551 [7]. The Node Energy Object can be used to specify the power source $(P S)$ of the node or compute the minimum battery level $(M B L)$ on the route. To support heterogeneous wireless M2M networks containing both BP nodes and MP nodes, we introduce two battery node aware path level routing metrics.

Battery-powered Node Count $(B N C)$ of a route is defined as the number of $\mathrm{BP}$ nodes on the route. This metric is used to discover routes containing fewer BP nodes. It shifts workload from BP nodes to MP nodes. During route discovery, destination node sets $B N C=0$. A BP node that forwards a route discovery message increases $B N C$ by 1 , a MP node does not modify the value of $B N C$.

Battery-powered Overhearing Count $(B O C)$ of a route counts the total number of times BP neighbors can overhear packet transmissions along the route. If a BP node is a neighbor of multiple nodes on the route, this BP node is counted multiple times. This metric is used to reduce battery energy wasted on overhearing. During route discovery, each node indicates if it is mains powered or battery powered. The source node sets $B O C$ to its $N o B P N$. At each hop, a node $N$ on the route updates $B O C$ as following:

- If node $N$ is battery powered, it decreases the received $B O C$ by 1 since previous hop must count node $N$ as a BP neighbor.

- If previous hop is battery powered, node $N$ decreases its $N o B P N$ by 1 since previous hop is actually on the route and will not suffer any additional energy loss due to its forwarding of the packet.

- $\quad$ Node $N$ then adds its updated $N o B P N$ to the updated $B O C$ and includes new $B O C$ in route discovery message.

\section{BATTERY ENERGY EFFICIENT RPL (B-RPL) FOR HETEROGENEOUS WIRELESS M2M NETWORKS USING Distributed SLEEP MODEL}

Using the newly introduced battery node aware routing metrics and existing RPL energy related routing metrics, this section presents a battery energy efficient RPL (B-RPL) to route packets in heterogeneous wireless M2M networks. BP nodes manage their sleep schedules based on the proposed distributed sleep model.

\section{A. Battery Node Aware Route Discovery}

Fig. 2 shows route discovery using the new battery node aware routing metrics and RPL defined metrics. When a node receives a DIO message for a new DODAG, it decides if it joins the DODAG. If yes, the DIO is processed. If $B N C=$ 0 , the node sets the DIO sender as its preferred parent and computes its rank. If $B N C \neq 0$, it selects DIO sender as a parent, but does not compute its rank. Instead, it starts a timer to wait for a more preferred DIO. If a node receives a DIO for an existing DODAG, it has already selected at least one parent. So, it checks if its rank is computed. If yes and it finds that the rank in DIO is greater than or equal to its current rank, the DIO is discarded. Otherwise, the node checks if its parent set is large enough. If no, it adds the DIO sender to its parent set, if yes, node updates its parent set by replacing one of the parents if this DIO contains a better route. If its rank is not computed and this DIO has a $B N C=0$, node sets the DIO sender as preferred parent, cancels DIO waiting timers, computes its rank and updates parent set by adding the DIO sender to its parent set if more parents are needed or replacing one of parents if parents are enough. If DIO has a $B N C>0$, the node adds DIO sender to its parent set if more parents are needed or otherwise updates its parent set if parents are enough. When DIO waiting timer expires, node selects one parent from parent set as preferred parent and computes its rank. A node schedules a DAO transmission, updates $B N C$ and $B O C$ metrics and transmits DIO message once it has selected its preferred parent. Besides conventional routing metrics, a node includes the updated $B N C$ and $B O C$ metrics in DIO transmission.

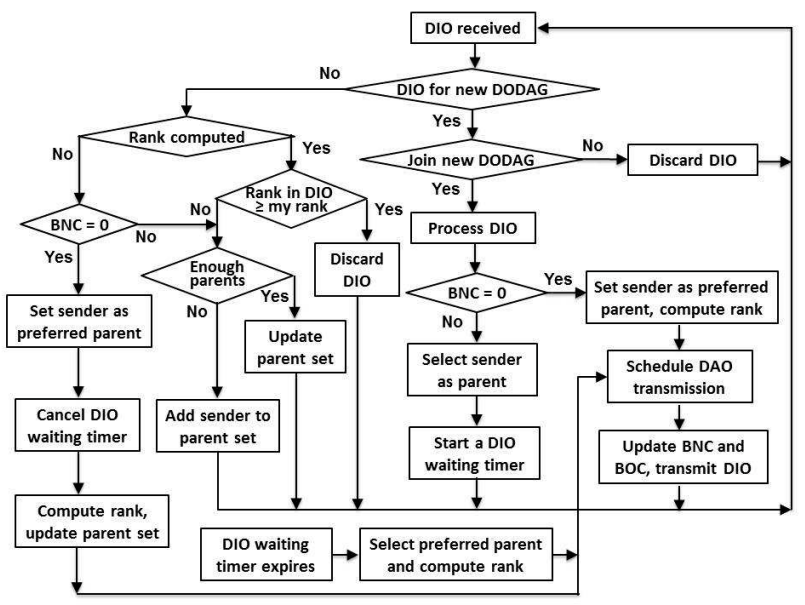

Fig. 2: Flow Chart of Route Discovery with New Routing Metrics

There are different ways to define whether one route is better than another route. We present a method to determine a better route. For each parent candidate $P_{c}$, a node calculates a parent qualification value $P_{q}\left(P_{c}\right)$ as follows.

$$
P_{q}\left(P_{c}\right)=\frac{B N C\left(P_{c}\right) \times B O C\left(P_{c}\right)}{\left(1+M B L\left(P_{c}\right)\right)}
$$


A smaller $P_{q}$ generally implies that the parent candidate provides a route with smaller number of BP nodes, smaller number of battery powered overhearing nodes and greater minimum battery level. Hence, it indicates a better route. When DIO waiting timer expires, a node selects a parent with the least $P_{q}$ as preferred parent. The $P S$ metric can be used to break tie.

\section{B. Battery Energy Efficient Data Packet Transmission and Forwarding}

In RPL, a node sends upward data packets to its preferred parent. However, the presence of the sleeping nodes challenges this approach. With the distributed sleep model, a node and its preferred parent may be awake at different times. The node can wait for its preferred parent to wake up, but idle listening consumes energy, causes packet drop due to full buffer and increases packet latency. Therefore, the routing algorithm must efficiently manage data packet transmission to avoid packet loss and energy waste. In B-RPL, a node can send data packets to any awake parent. If no parent is awake, the node may go to sleep or wait based on the proposed sleep management solution. If multiple parents are awake, the node selects an awake parent that provides a route with the minimum battery energy consumption. This section presents a method to determine the most battery energy efficient parent.

We denote battery energy consumption on idle listening for wakeup message, wakeup message transmission, wakeup message receiving, data packet transmission and data packet receiving as $P_{i}, W_{t}, W_{r}, P_{t}$ and $P_{r}$, respectively. In addition, BP neighbors also consume their energy on overhearing, which can cost $20 \%$ - 30\% more energy than idle listening [8]. A BP node overhears neighbor's transmission if it is awake. The probability of a BP node $B$ being awake is $P_{a}(B)=$ $|A P(B)| /(|A P(B)|+|S P(B)|)$.

We first compute battery energy consumption to transmit a data packet from one node to another node. Depending on the power sources of transmitter and receiver, there are four cases of battery energy consumption: $E\left(B_{t}, B_{r}\right), E\left(M_{t}, B_{r}\right)$, $E\left(B_{t}, M_{r}\right)$ and $E\left(M_{t}, M_{r}\right)$, where $B_{t}, B_{r}, M_{t}$ and $M_{r}$ denote the BP transmitter, the BP receiver, the MP transmitter and the MP receiver, respectively. In equations (3) to (6), $N_{t}$ denotes number of the BP neighbors of the transmitter and $N_{r}$ denotes number of the BP neighbors of the receiver.

$$
\begin{aligned}
& E\left(B_{t}, B_{r}\right)=P_{i}\left(B_{t}\right)+W_{t}+W_{r}+P_{t}+P_{r} \\
& +\sum_{k=1}^{N_{r}} P_{a}\left(B B_{r}^{k}\right) \times W_{r}+\sum_{j=1}^{N_{t}} P_{a}\left(B B_{t}^{j}\right) \times P_{r}
\end{aligned}
$$

where $N_{r}$ excludes $B_{t}, B B_{r}^{k}\left(k=1\right.$ to $\left.N_{r}\right)$ are $B_{r}$ 's BP neighbors excluding $B_{t}, N_{t}$ excludes $B_{r}$ and $B B_{t}^{j} \quad(j=1$ to $N_{t}$ ) are $B_{t}$ 's BP neighbors excluding $B_{r}$.

$$
\begin{aligned}
E\left(M_{t}, B_{r}\right)=W_{t}+P_{r} & +\sum_{k=1}^{N_{r}} P_{a}\left(B B_{r}^{k}\right) \times W_{r} \\
& +\sum_{j=1}^{N_{t}} P_{a}\left(B M_{t}^{j}\right) \times P_{r}
\end{aligned}
$$

where $B B_{r}^{k}\left(k=1\right.$ to $\left.N_{r}\right)$ are $B_{r}$ 's BP neighbors, $N_{t}$ excludes $B_{r}$ and $B M_{t}^{j}\left(j=1\right.$ to $\left.N_{t}\right)$ are $M_{t}$ 's BP neighbors excluding
$B_{r}$.

$$
E\left(B_{t}, M_{r}\right)=P_{t}+\sum_{j=1}^{N_{t}} P_{a}\left(B B_{t}^{j}\right) \times P_{r}
$$

where $B B_{t}^{j}\left(j=1\right.$ to $\left.N_{t}\right)$ are $B_{t}$ 's BP neighbors.

$$
E\left(M_{t}, M_{r}\right)=\sum_{j=1}^{N_{t}} P_{a}\left(B M_{t}^{j}\right) \times P_{r}
$$

where $B M_{t}^{j}\left(j=1\right.$ to $\left.N_{t}\right)$ are $M_{t}$ 's BP neighbors.

The next step is to compute battery energy consumption $E(S, D)$ by transmitting a data packet from the source node $S$ to the destination node $D$. On a route from $S$ to $D$, assume $B 2 B$ transmissions from $B_{t}$ to $B_{r}, M 2 B$ transmissions from $M_{t}$ to $B_{r}, B 2 M$ transmissions from $B_{t}$ to $M_{r}$, and $M 2 M$ transmissions from $M_{t}$ to $M_{r}$. Using equations (3) to (6), the $E(S, D)$ is given by

$$
\begin{aligned}
E(S, D) & =\sum_{i=1}^{B 2 B} P\left(B_{t}^{i}, B_{r}^{i}\right)+\sum_{j=1}^{M 2 B} P\left(M_{t}^{j}, B_{r}^{j}\right) \\
+ & \sum_{k=1}^{B 2 M} P\left(B_{t}^{k}, M_{r}^{k}\right)+\sum_{l=1}^{M 2 M} P\left(M_{t}^{l}, M_{r}^{l}\right)
\end{aligned}
$$

The third step is to compute the maximum and the minimum values of $E(S, D)$. On a given route, the layout of nodes affects the battery energy consumption. Including $S$ and $D$, assume the given route has $N_{B} \mathrm{BP}$ nodes $B_{1}, B_{2}, \ldots, B_{N_{B}}$ and $N_{M}$ MP nodes $M_{1}, M_{2}, \ldots, M_{N_{M}}$. Equations (3) to (6) indicate that the maximum battery energy consumption occurs when all MP nodes are on the first portion of the route followed by all BP nodes.

$$
\begin{aligned}
& E(S, D)_{\max }=\sum_{j=1}^{N_{M}-1} P\left(M_{t}^{j}, M_{r}^{j}\right)+P\left(M_{t}, B_{r}\right) \\
& +\sum_{i=1}^{N_{B}-1} P\left(B_{t}^{i}, B_{r}^{i}\right)
\end{aligned}
$$

The minimum battery energy consumption occurs when BP nodes and MP nodes are interleaved.

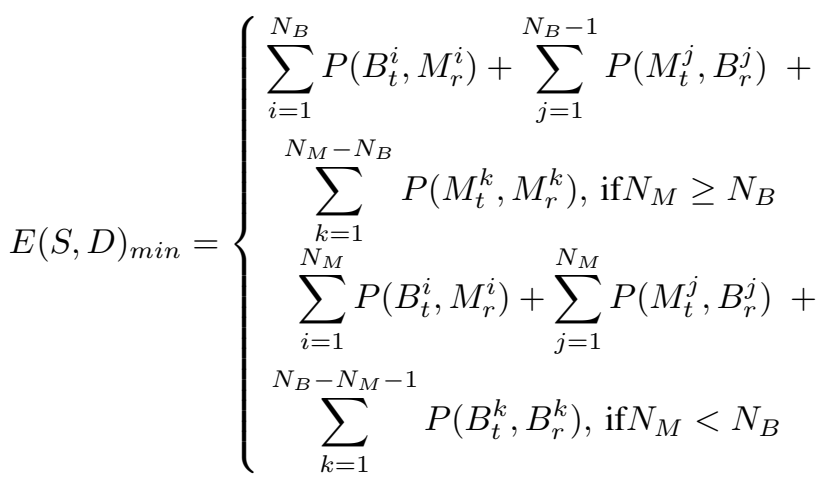

Using the $E(S, D)_{\max }$ and the $E(S, D)_{\min }$, the average battery energy consumption by transmitting a data packet from the source node $S$ to the destination node $D$ is given by

$$
E(S, D)_{a v g}=\frac{E(S, D)_{\max }+E(S, D)_{\min }}{2}
$$


Therefore, if there are multiple parents awake, a source node $S$ shall forward data packets to an awake parent with the minimum $E(S, D)_{\text {avg }}$.

To compute $E(S, D)_{a v g}$ in practice, we need to know the number of MP nodes $(N o M P N)$, the $N o B P N$ for each node on the route and the probability of each $\mathrm{BP}$ neighbor being awake. $N o M P N=H C-B N C+1$, where $H C$ is the hop count. So, the total number of nodes is $N o M P N+B N C$. On average, each node has $B O C /(N o M P N+B N C) \mathrm{BP}$ overhearing neighbors. To compute $P_{a}(B)$, a typical AP length and SP length can be used to estimate the probability of a battery powered neighbor being awake.

\section{Performance Evaluation}

In this section, we present performance evaluation of the BRPL using the NS2 simulator. The standard RPL is used as the benchmark for performance comparison. We simulated a heterogeneous wireless network containing 500 nodes deployed in a $22 \times 23$ grid. Each unit in the grid represents a $10 \times 10$ square meter field and contains a single node randomly placed in the unit. BP nodes and MP nodes are randomly selected. The percentage of MP nodes varies. A data sink is placed at the center of the grid. IEEE 802.15.4 MAC and PHY are modified for sleep support. All BP nodes have $100 \%$ of battery level at the begining of simulation. The sleep interval for all BP nodes is 100 seconds with 1 second of active period and 99 seconds of sleep period. The buffer size of each node is 15. Each node sends a data packet to the sink node every 100 seconds. The simulation runs 10000 seconds. Following figures show simulation results for $50 \%$ of MP nodes.

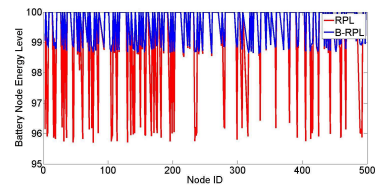

Fig. 3: Battery Node Energy Level

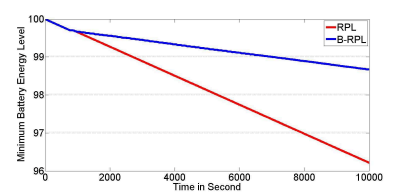

Fig. 4: Minimum Battery Energy
Fig. 3 shows residual battery level of the BP nodes. On average, RPL consumes nearly two times more battery energy than B-RPL does. This is because a RPL node always sends data packets to its preferred parent, which causes longer idle time. On the other hand, a B-RPL node can send data packets to any awake parent and selects an awake parent that provides a route with the least battery energy consumption. In addition, B-RPL discovers more battery energy efficient routes. Fig. 4 shows the minimum residual battery level among all BP nodes, which indicates that in 10000 seconds of simulation, the maximum battery energy consumption for B-RPL is $1.3 \%$ and the maximum battery energy consumption for RPL is $3.8 \%$. It means that B-RPL can run network for 213.7 hours and RPL can only run network for 73.1 hours. Therefore, B-RPL extends network lifetime by 1.92 times.

Fig. 5 shows data packet delivery rate (DPDR). B-RPL improves the DPDR by $63 \%$ from $35 \%$ to $98 \%$. B-RPL obtains $70 \%$ of DPDR at network startup. Once network construction completes, B-RPL achieves a steady $98 \%$ of DPDR. For RPL, the packet drop is mainly caused by buffer overflow. Fig. 6 shows the CDF distribution of data packet delay. B-RPL

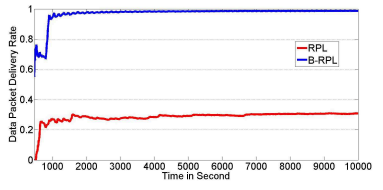

Fig. 5: Data Packet Delivery Rate

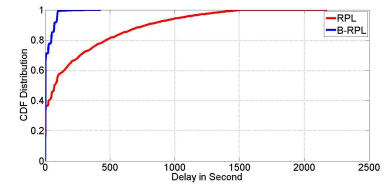

Fig. 6: Data Packet Delay delivers $99 \%$ of data packets in 100 seconds and RPL only delivers $55 \%$ of data packets in 100 seconds. B-RPL reduces average data packet delay (ADPD) by $92.6 \%$ from 243 seconds to 18 seconds and the maximum data packet delay (MDPD) by $80 \%$ from 2174 seconds to 427 seconds.

For $30 \%$ of MP nodes, B-RPL increases network lifetime by $26 \%$ from 69.4 hours to 87.4 hours, improves DPDR by $75 \%$ from $12.5 \%$ to $87.5 \%$ and reduces ADPD by $90.9 \%$ from 275 seconds to 25 seconds. For $70 \%$ of MP nodes, B-RPL extends network lifetime by 2 times from 73.5 hours to 221.1 hours, improves DPDR by $20.7 \%$ from $78.6 \%$ to $99.3 \%$ and reduces ADPD by $87.4 \%$ from 87 seconds to 11 seconds.

\section{CONCLUSION}

Battery powered devices will play important role in wireless M2M networks. For such networks, saving energy and extending the lifetime of battery powered nodes become critical. In this paper, we present battery energy management solutions for heterogeneous wireless M2M networks containing battery powered nodes and mains powered nodes. We propose a distributed sleep model for battery powered nodes to dynamically manage their sleep schedules based on their internal parameters and observed network conditions. We also propose a battery energy efficient routing protocol called BRPL to enhance standard RPL for sleep operation support in battery operated networks that use distributed sleep control. Simulation results show that B-RPL outperforms RPL in terms of data packet delivery rate, data packet delay and the network lifetime extension. B-RPL can improve data packet delivery rate by $75 \%$, reduce average data packet delay by $92.6 \%$ and extend the network lifetime by two times.

\section{REFERENCES}

[1] A. A. Aziz, Y. A. Sekercioglu, P. Fitzpatric, and M. Ivanovich, "A Survey on Distributed Topology Control Techniques for Extending the Lifetime of Battery Powered Wireless Sensor Networks," in IEEE Communications Survey and Tutorials. IEEE, 2013, pp. 121-144.

[2] M. Khan, W. N. Gansterer, and G. Haring, "Congestion Avoidance and Energy Efficient Routing Protocol for Wireless Sensor Networks with a Mobile Sink," Journal of Networks, , vol. 2, no. 6, 2007.

[3] T. Winter and et al, "RFC 6550," http://tools.ietf.org/html/rfc6550.

[4] E. Ancillotti, R. Bruno, and M. Conti, "The Role of the RPL Routing Protocol for Smart Grid Communications," Communications Magazine, IEEE, vol. 51, no. 1, pp. 75-83, 2013.

[5] B. Pavkovic, F. Theoleyre, and A. Duda, "Multipath Opportunistic RPL Routing over IEEE 802.15.4," in MSWiM'11. ACM, 2011.

[6] X. Yang, J. Guo, P. Orlik, K. Parsons, and K. Ishibashi, "Stability Metric Based Routing Protocol for Low-Power and Lossy Networks," in Proceedings of International Conference on Communications (ICC), 2014 IEEE. IEEE, 2014.

[7] J. Vasseur and et al, "RFC 6551," http://tools.ietf.org/html/rfc6551.

[8] B. Krishnamachari, Networking Wireless Sensors. Cambridge, UK: Cambridge University Press, 2005. 